\title{
SISTEMI CONIUGATI SULLE SUPERFICIE DEGLI IPERSPAZI.
}

\author{
Memoria di Enrico Bompiani (Roma).
}

Adunanza del 27 novembre 1921.

S

\section{Introduzione.}

Lo studio delle curve definite sopra una superficie da proprietà proiettivo-differenziali ha sopratutto lo scopo di fornire una interpretazione geometrica delle equazioni lineari ed omogenee a derivate parziali. In questo senso è stata già ampiamente studiata l'equazione di LAPLACE ${ }^{\mathbf{I}}$ )

$$
\frac{\partial^{2} x}{\partial \tau_{1} \partial \tau_{2}}+a\left(\tau_{1}, \tau_{2}\right) \frac{\partial x}{\partial \tau_{1}}+b\left(\tau_{1}, \tau_{2}\right) \frac{\partial x}{\partial \tau_{2}}+c\left(\tau_{1}, \tau_{2}\right) x=0
$$

le cui soluzioni (in numero qualsiasi) possono venir interpretate come coordinate proiettive omogenee dei punti di una superficie che possiede un doppio sistema coniugato di curve nel senso ordinario. Della necessità di introdurre un modello iperspaziale nello studio di quella equazione ci si rende facilmente conto se si ricorda che, mentre nello spazio ordinario ogni superficie ammette infiniti sistemi coniugati, una superficie dello $S_{4}$ ammette un solo sistema coniugato, e una superficie di uno spazio di dimensione superiore non ne ammette alcuno in generale: quindi le superficie che ne posseg. gono uno, rappresentano un'eccezione dotata di proprietd sue peculiari. E sono queste proprietà geometriche che vengono interpretate a vantaggio della teoria analitica dell'equazione di LAPLACE.

Però questo stesso vantaggio menoma in un certo senso l'interesse di quei sistemi di curve; perchè non esistendo sopra una superficie generale di un iperspazio non ci

1) Puó vedersi in proposito la mia Memoria: Sullequazione di LAPLACE [Rendiconti del Circolo Matematico di Palermo, t. XXXIV (20 semestre 19I2), pp. 383-407]. 
informano affatto sulla sua natura. Occorre perciò ricercare altri sistemi di curve definiti da proprietà simile a quella che definisce i sistemi coniugati; e su di essi si potrà costruire una teoria analoga a quella cui si è precedentemente accennato. Questi sistemi esistoro realmente e ne ho gid mostrato incidentalmente l'interesse in un problema di natura metrica ${ }^{2}$ ); questa Nota è destinata sopratutto a mettere in evidenza le analogie e le differenze dal punto di vista proiettivo, con i sistemi coniugati ordinari.

I sistemi di curve qui studiati (come altri che si possono immaginare, per es. quelli di quasi-asmtotiche ai quali ho dedicato altri lavori) essendo invarianti per trasformazioni proiettive forniscono lo strumento naturale, e verranno certamente utili, per approfondire la bella teoria delle applicabilita proiettive per le superficie iperspaziali che dobbiamo al prof. G. Fubini.

\section{S2.}

\section{Posizione congiunta dei piani tangenti ad una superficie di $S_{n}$.}

Due piani tangenti ad una superficie $V_{2}$ di $S_{n}$ in due punti infinitamente vicini hanno sempre un punto comune: questo fatto si esprime dicendo che $\mathrm{i}$ due piani sono in posizione congiunta (vereinigte Lage secondo LIE). La dimostrazione è immediata. Ne segue che se si considera un elemento $E_{s}$ di una curva su $V_{2}$ (cioè un punto di $V_{2}$ e gli elementi della curva contenuti nell'intorno d'ordine $s$ del punto) i piani tangenti a $V_{2}$ nei punti di $E_{s}$ appartengono a uno spazio di dimensione $\leq 2 s+2$ e varrà necessariamente il segno di disuguaglianza se $2 s+2>n$.

\section{$\$ 3$.}

\section{Definizione dei sistemi coniugati di specie $\vee$ sulle superficie di $S_{2 v+1}$.}

Sopra una $V_{2}$ di $S_{2 v+1}$ consideriamo un elemento $E_{v}$ di curva $C$ passante regolarnente per un suo punto $P \equiv E_{\mathrm{o}}$; i piani tangenti a $V_{2}$ nei punti di $E_{\mathrm{v}}$ successivi a $P$ determinano un $S_{2 y}$ che taglia il piano tangente in $P$ lungo una retta uscente da $P$. Questa tangente $\left(E_{1}\right)$ in $P$ si diri tangente coniugata di specie $\vee$ dell'elemento $E_{\nu}$.

Se consideriamo poi un sistema $\infty^{\mathrm{I}}$ di curve $C$ arbitrariamente date su $V_{2}$, e per ogni punto $P$ costruiamo la tangente coniugata (nel senso ora specificato) all'elemento

2) E. Bompiant, Problemi nuovi di geometria metrico-differenziale [Rendiconti della R. Accademia dei Lincei, serie V, vol XXIV (I semestre I915), pp. I193-I199]. 
$E_{v}$ di curva $C$ che vi passa, le tangenti costruite inviluppano su $V_{2}$ un nuovo sistema $\infty^{\prime}$ di curve che diremo sistema coniugato di specie $\vee$ al sistema delle $C$.

Indicheremo una curva di questo nuovo sistema con $K$, e per ricordare che del primo sistema occorre considerare gli elementi $E_{v}$, del secondo gli elementi $E_{1}$, adottiamo la notazione $C^{(v)}$ e $K^{(\mathrm{r})}$.

I due sistemi insieme formano il doppio sistema coniugato di specie $\vee\left(C^{(\nu)}, K^{(x)}\right)$.

Per $\nu=I$ si hanno $i$ sistemi coniugati ordinari sulle $V_{2}$ di $S_{3}$; per $\nu>$ I si perde in generale il carattere involutorio, non essendo lecito lo scambio delle $C^{(\text {(v) }}$ con le $K^{(1)}$.

\section{$\$ 4$.}

\section{Proprietà caratteristica della rigata delle tangenti coniugate agli elementi $E_{\nu}$ di una curva.}

Ho già introdotto, per la classificazione proiettiva delle rigate di un $S$, la nozione di (primo) indice di sviluppabilita ${ }^{3}$ ): esso è il massimo numero di generatrici consecutive linearmente indipendenti e raggiunge per una rigata generale di $S_{r}$, i valori $\frac{r}{2}$ o $\frac{r+I}{2}$ secondo la parità di $r$.

La proprietà caratteristica della rigata indicata è la seguente: Le tangenti coniugate di specie $\vee$ agli elementi $E_{y}$ di una curva di $V_{2}$ formano una rigata d'indice di sviluppabilità $\vee$ (invece che $\vee+1$ ) e viceversa.

La parte diretta del teorema segue senz'altro dalla costruzione delle tangenti coniugate; l'inversa dal fatto che se la rigata è d'indice $v$, lo $S_{2 \nu}$ di $v+1$ generatrici consecutive contiene $\mathrm{i}$ piani tangenti in $\vee$ punti successivi delle curve di contatto ${ }^{4}$ ).

Limiteró d'ora innanzi lo studio ai sistemi coniugati di $2^{2}$ specie.

3) E. Bompiani, Alcune proprietd proiettivo-differenziali dei sistemi di rette negli iperspazi [Rendiconti del Circolo Matematico di Palermo, t. XXXVII ( ${ }^{0}$ semestre 1914), pp. 305-331].

4) Si puó domandare se, e in qual modo, si possa estendere ai sistemi coniugati con $\nu>I$ il teorema di Konnigs che fornisce $\infty^{4}$ sistemi coniugati sopra una superficie di $S_{3}$. S1 prevede che qua. lora ció sia possibile per una superficie $a$ di $S_{2 y+1}$, lo sarà in due modı diversi in ragione del diverso comportamento delle curve $C$ e $K$ nel conugio per $\nu>1$.

E si hanno infatti $i$ due risultati seguenti :

Se $i$ pian tangenti a $\sigma$ incontrano tutti uno stesso $S_{\nu}$ si conoscono su di essa $i$ sistemi $\left(C^{(v)}, K^{(1)}\right)$ costituiti :

$\mathrm{I}^{\circ}$ ) dalle $\infty^{\mathrm{l}}$ curve $K$ (certo esistenti su $\sigma$ ) in $\infty^{\mathrm{t}} S_{\nu+1}$ passanti per $S_{\nu}$;

$\left.2^{\circ}\right) d a \infty^{\mathrm{r}}$ fra le $\infty^{\nu}$ curve $C d t$ contatto delle rigate circoscritte a $\sigma$ e aventi ciascuna un $S_{\nu-1}$ direttore (cioe con generatrici incidenti un $S_{\nu-1}$ ) contenuto in $S_{\nu}$.

Il procedimento indicato, valido per $\sigma$ particolari appena $\nu>1$, fornisce tutte le $C$ che associate 
$\$ 5$.

Equazione differenziale dei sistemi coniugati di $2^{a}$ specie.

Sulla superficie $V_{2}$ descritta dal punto $x\left(\tau_{1}, \tau_{2}\right)$ [cioè di coordinate proiettive omogenee $x_{i}\left(\tau_{1}, \tau_{2}\right)$ con $\left.i=0, \mathrm{I}, \ldots, 5\right]$ sia definito il sistema $\infty^{2}$, di curve $C^{(2)}$ (anche differenzialmente): sarà noto per ogui punto il valore di $d \tau_{1} / d \tau_{2}$ relativo alla $C^{(2)}$ che vi passa. Indichiamo con $\delta$ le caratteristiche dei differenziali relativi alle curve $K^{(s)}$ del sistema coniugato.

La condizione di coniugio è data dall'equazione determinante (di cui si indica una colonna generica omettendo l'indice $i$ delle $x$ ):

$$
\left|\begin{array}{c}
x \\
x^{\mathrm{oO}} \\
x^{0 \mathrm{1}} \\
x^{20} d \tau_{\mathrm{r}}+x^{11} d \tau_{2} \\
x^{11} d \tau_{\mathrm{r}}+x^{02} d \tau_{2} \\
\left(M+x^{20} d^{2} \tau_{\mathrm{r}}+x^{11} d^{2} \tau_{2}\right) \delta \tau_{1}+\left(N+x^{11} d^{2} \tau_{1}+x^{02} d^{2} \tau_{2}\right) \delta \tau_{2}
\end{array}\right|=0
$$

ove

$$
\begin{gathered}
x^{+s}=\frac{\partial^{1+s} x}{\partial \tau_{1}^{r} \partial \tau_{2}^{s}} \\
M=x^{30} d \tau_{1}^{2}+2 x^{2 \mathrm{I}} d \tau_{1} d \tau_{2}+x^{12} d \tau_{2}^{2} \\
N=x^{21} d \tau_{1}^{2}+2 x^{12} d \tau_{1} d \tau_{2}+x^{03} d \tau_{2}^{2} .
\end{gathered}
$$

Se i sistemi $K^{(1)}$ e $C^{(2)}$ sono distinti con opportuna scelta delle linee coordinate (cioè facendo $d \tau_{2}=0$ su $C^{(2)}$ e $\delta \tau_{1}=0$ su $K^{(1)}$ ), il coniugio di $2^{2}$ specie equivale

alle $\infty^{1} K$ dànno sistemi $\left(C^{(v)}, K^{(1)}\right)$ d'accordo col fatto che, date le $K$, l'equazione differenziale che determina le $C$ è d'ordine v.

Per una $\sigma$ generica si ha l'altro risultato:

St consideri, insieme a $\sigma$ di $S_{2 \nu+1}$, la superficie $\bar{\sigma}$ hoogo delle traccie dei suoi piani tangenti sopra un $S_{2 \nu \ldots}$ : si ba cosi una corrispondenza fra punti (generici) di $\sigma e \bar{\sigma}$. Al sistema delle $\infty^{\top}$ sezioni $C$ pro. dotte su $\sigma$ dagli iperpiani per $S_{2 \nu-1}$ corrisponde un sistema $\infty^{\mathrm{I}}$ di curve $\bar{C}$ su $\bar{\sigma}$; sia $\bar{K}$ il sistema coniugato di specie $\vee-$ I I $_{\text {I }} \bar{\sigma}$ alle $\bar{C}$ in modo da avere un doppio sistema $\left.\overline{(C}^{(\nu-1)}, \vec{K}^{(1)}\right)$. Le curve $K$ di $\sigma$ che corrispondono alle $\bar{K}$ di $\bar{\sigma}$ formano con le $C$ un doppio sistema coniugato di specie $\vee\left(C^{(\hat{})}, K^{(1)}\right)$.

Si sanno co.l costruire $0^{4 \nu}$ sistemi coniugati di specie $\nu$ appena si sappiano costruire $i$ sistemi coniugati di specie $v-1$ per una superficie di $S_{2 v-1}: s_{1}$ ha cioe una riduzione, per essi, della difficoltà d'integrazione (come nel teorema di Koentas); però il procedimento non è applicabile in modo ricorrente, perchè le $\bar{C}$ su $\bar{\sigma}$ non presentano la particolatità che presentavano le $C$ su $\sigma$. 
all'esistenza di un'cquazione a derivate parziali

$$
x^{25}+a x^{20}+b x^{11}+c x^{10}+d x^{01}+e x=0
$$

$\left(a, \ldots, e\right.$ funzioni di $\left.\tau_{1}, \tau_{2}\right)$ soddisfatta da tutte le $x_{i}$ : questa equazione $\dot{e}$, per il coniugio di $2^{a}$ specie, l'analoga dell'equazione di LAPLACE, ricordata per il coniugio ordinario.

Se invece i sistemi $C^{(2)}$ e $K^{(1)}$ coincidono, quindi $d=\delta$ la (I) diviene:

$$
\left|\begin{array}{c}
x \\
x^{10} \\
x^{01} \\
x^{20} d \tau_{1}+x^{11} d \tau_{2} \\
x^{11} d \tau_{1}+x^{02} d \tau_{2} \\
x^{30} d \tau_{1}^{2}+3 x^{2 \mathrm{r}} d \tau_{1}^{2} d \tau_{2}+3 x^{12} d \tau_{1} d \tau^{2}+x^{0 ;} d \tau_{2}^{3}
\end{array}\right|=0 .
$$

Questa equazione definisce in ciascun punto le cinque tangenti principali, e sulla superficie le linee principali ${ }^{5}$ ): esse appariscono qui come linee autoconiugate di $\mathbf{2}^{a}$ specie; è questa una loro nuova proprietà caratteristiçą.

\section{$\$ 6$.}

\section{Influenza di un sistema coniugato ordinario sopra quelli di specie superiore.}

Una superficie di $S_{5}$ non possiede in generale un doppio sistema coniugato ordinario $(\nu=I)$ nè un sistema semplice di asintotiche.

5) C. Segre, Prelininarı di una teoria delle varieta luoghi di spazı [Rendiconti del Circolo Matematico di Palermo, t. XXX (2 ${ }^{\circ}$ semestre I910), pp. 87-121].

Per un'altra proprietà di esse vedi la mia Nota Sopra alcune estensioni dei teoremi di Meusnier $e d \imath$ Eulero [Atti della R. Accademia delle Scienze di Torno, t. XLVIII (19I2-19I3), pp. 393.4Io]: secondo la denominazione ivi introdotta, esse costituiscono una (particolare) famiglia di quasi-asintotiche $\gamma_{2, j}$, perchè lo $S_{3}$ osculatore ad una di esse in un punto coincide con lo $S_{3}$ 2-osculatore alla superficie secondo la tangente ivi alla curva stessa.

Il Prof SEgre ha poi trovato recentemente che l'indeterminazione delle linee principali è proprietà caratteristica delle superficie di Veronese. Vedi SEGRE, Le linee principali ăi una superficie di $S_{5}$ e una proprietd caratteristica della superficie dt VERONese [Rendiccinti della R. Accademid dei Lincei, serie V, vol. XXX ( ${ }^{\circ}$ semestre I92I), pp. 200-203, 227-23I]. Una proprietà caratteristica delle superficie che rappresentano la totalità delle curve piane di dato ordine, dipendente dell'esistenza di linee quasi-asintotiche si trova nella mia Nota: Proprietd differenziale caratteristica delle superficie che rappresentano la totalità delle curve piane algebriche di dato ordine [Rendiconți della $R$. Accadęmia dei Lincei ( ${ }^{\circ}$ semestre $192 \mathrm{I}$ ), in corso di stampa]. 
Vogliamo cercare come l'esistenza di uno dl questi sistemi modifichi i risultati relativi al coniugio di $2^{\mathrm{a}}$ specie.

Questi due fatti possono raccogliersi dicendo semplicemente che lo spazio 2-osculatore in un punto generico $x^{6}$ ), individuato dai punti $x, x^{\text {to }}, x^{01}, x^{20}, x^{1 \mathrm{r}}, x^{\mathrm{or}}$ è uno $S_{4}$. Esiste in tal caso un'equazione lineare omogenea a derivate parziali, del $2^{\circ}$ ordine, cui soddisfano le coordinate $x_{i}$. In forza di essa l'equazione (I) che esprime il co. niugio di $2^{2}$ specie si riduce al tipo

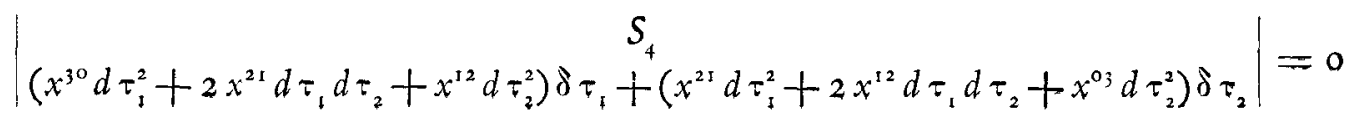

avendo globalmente indicato con $S_{f}$ le prime $S$ linee costruite con le coordinate dei punti che individuano lo $S_{4}$.

Apparisce da questa equazione una differenza essenziale fra questo caso e quello generale: perche fissata la tangente $d$ (relativa a una $C$ ) rimane fissata una tangente $\delta$, ma fissata $\delta$ si hanno due direzioni $d$; cioè mentre nel caso generale dato un sistema $K^{(1)}$ si possono costruire $\infty^{2} C$ tali che $\infty^{\mathrm{i}}$ di esse formino con $K^{(\mathrm{r})}$ un doppio sistema coniugato, nel caso attuale dato un sistema $K^{(1)}$ esistono due soli sistemi $C$ ad esso coniugati.

La proprietd rilevata s'inverte. Supponiamo che ad ogni sistema di curve $K^{(3)}$ si possa far corrispondere un numero finito di sistemi $C$ : dico che lo $S(2)$ osculatore generico alla superficie è un $S_{4}$.

Infatti in questa ipotesi l'equazione ( $\mathrm{I}$ ) deve essere di $\mathrm{I}^{\circ}$ ordine nei differenziali, cioè qualunque sia $d \tau_{1} / d \tau_{2}$, devono essere nulli $\mathrm{i}$ coefficienti di $d^{2} \tau_{1}, d^{2} \tau_{2}$. Se si scrive questo si ottiene appunto un'equazione a derivate parziali del $2^{\circ}$ ordine, lin. ed omog., cioè $S(2) \equiv S_{4}$. Si puó osservare che lo stesso ragionamento vale qualunque sia l'ambiente della superficie (purchè questa possegga doppi sistemi coniugati di $2^{2}$ specie); sicchè :

Condizione necessaria e sufficiente affincbe una superficie di $S_{n}(n \gg 5)$ abbia come spario 2-osculatore generico uno $S_{4}\left(e\right.$ non $\left.S_{5}\right)$ i che ad ogni sistema $K^{(1)}$ di curve si possa associare un numero finito (necessariamente I o 2) di sistemi di curve in modo da formare un doppio sistema conilugato di $2^{a}$ specie.

Se chiamiamo ancora principali le tangenti autoconiugate (per le quali $d=\delta$ ) troviamo che da ogni punto della superficie ne escono tre; le superficie a tangenti principali indeterminate stanno in $S_{4}$.

6) Per la definizione degli spazi v-osculatori, $S(v)$, puó vedersi la niia Nota: Sopra alcune estenstoni, etc. già citata in 5 ). 


\section{Superficie che posseggono un sistema di curve per le quali è indeterminato il sistema coniugato.}

Se $v=1$ si hanno le sviluppabili (in particolare il piano).

Se $v=2$ bisogna distinguere due tipi di superficie, secondo che su di essa:

$\mathrm{I}^{\circ}$ ) Esiste un sistema $C$ per il quale $e$ indeterminato il sistema $K$.

$\left.2^{\circ}\right)$ Esiste un sistema $K$ per il quale è indeterminato il sistema $C$.

Nel $I^{\circ}$ caso: assumiamo le $C$ date come çurve $\tau_{z}\left(\tau_{f}=\right.$ cost.). L'equazione ( $\mathrm{I}$ ) che si riduce alla seguente

$$
\left|\begin{array}{c}
x \\
x^{10} \\
x^{\text {oI }} \\
x^{11} \\
x^{02} \\
x^{12} \delta \tau_{1}+x^{03} \delta \tau_{2}
\end{array}\right|=0
$$

deve dare un valore indeterminato per il rapporto $\delta \tau_{1} / \delta \tau_{2}$, sicibè esistono due equazioni del tipo

$$
\begin{aligned}
& x^{12}=\left[x^{11}, x^{02}, x^{10}, x^{01}, x\right] \\
& x^{03}=\left[x^{11}, x^{02}, x^{10}, x^{01}, x\right]
\end{aligned}
$$

intendendo con la notazione a $2^{\circ}$ membro una combinazione lineare omogenea delle quantità indicate, $i$ cui coefficienti sono funzioni (che non interessa di considerare) di $\tau_{1}, \tau_{2}$. Le coordinate dei punti della superficie soddisfano a queste equazioni e alle loro conseguenze differenziali. Possiamo anche dire che i punti indicati nei primi membri stanno nello $S_{4}$ dei punti indicati nei $2^{1}$ membri.

Derivando rispetto a $\tau_{2}$ la seconda equazione ricaviamo che tutti $i$ punti derivati $x^{\text {or }}$ con $r \gg 3$ stanno nello $S_{4}$ indicato, cioè ogni curva $C$ sta in $S_{4}$. Ma dalla prima risulta pure che tutti $i$ punti $x^{1 r}(r \supseteq 2)$ stanno nello $S_{4}$ il quale contiene quindi due curve $C$ infinitamente vicine. Se non tutta la superficie sta in $S_{4}$, caso che poco interessa, le curve $C$ apparterranno al più ad $S_{3}$ e due $S_{3}$ infinitamente vicini stanno in uno $S_{4}$ cioé si tagliano in $S_{2}$.

Rimane solo da esaminare il caso in cui valga una relazione del tipo

$$
\left[x^{11}, x^{02}, x^{10}, x^{01}, x\right]=0 ;
$$

con una trasformazione di variabili l'equazione precedente è sempre riducibile ad una delle due forme (senza alterare le linee $d \tau_{1}=0$ )

$$
x^{11}=\left[x^{10}, x^{01}, x\right] ; \quad x^{02}=\left[x^{10}, x^{01}, x\right]
$$


quindi le $C$ o fanno parte dı un sistema coniugato ordinario o sono asintotiche. Osservando che nulla cambia se la superficie sta in $S_{n}(n \supseteq S)$ purchè possegga la proprieti voluta, abbiamo:

Se una superficie di $S_{\text {u }}$ possiede un sistema di curve $C$ di cui sia indeterminato il sistema coningato di $2^{\text {a }}$ specie $K$ si presenta necessariamente uno dei casi seguenti:

I) La superficie sta in $S_{+}$.

2) Il sistema $C$ appartiene ad un doppio sistema coniugato ordinario o è composto di asintotiche.

3) Le curve $C$ sono in $S_{3}$ di cui due infinitamente vicini s'incidono in un piano; sicche o questi $S$; inviluppano una curva (caso generale) oppure (casi particolari) sono tangenti ad un cono di $2^{a}$ specie (con retta-vertice) o osculatori a un cono di $I^{a}$ specie (cioé contenenti tre generatrici successive) o infine passano tutti per uno stesso piano.

Esaminiamo ora l'altro caso: esiste sulla superficie un sistema di linee $K$ (che assumeremo come $\delta \tau_{2}=0$ ) di cui è indeterminato il sistema coniugato di $2^{2}$ specie $C$.

Ciò significa che posto nella (I) $\delta \tau_{2}=0$, l'equazione è identicamente soddisfatta quali si siano i valori attribuiti ai differenziali primi e secondi di caratteristica $d$ delle $\tau$.

Dall'annullarsi i coefficienti dei differenziali secondi si trae un'equazione del tipo:

$$
\left[x^{20}, x^{11}, x^{02} ; x^{10}, x^{01}, x\right]=0
$$

sicchè lo spazio 2-osculatore in un punto generico della superficie è uno $S_{4}$ (e non $S_{5}$ ).

Ciò fatto rimane da esprimere che è

cioè

$$
\left|\begin{array}{c}
S_{4} \\
x^{30} d \tau_{1}^{2}+2 x^{21} d \tau_{1} d \tau_{2}+x^{12} d \tau_{2}^{2}
\end{array}\right| \equiv 0
$$

$$
\left\{\begin{array}{l}
x^{30}=\left[S_{4}\right] \\
x^{21}=\left[S_{4}\right] \\
x^{12}=\left[S_{4}\right]
\end{array}\right.
$$

indicando con questa notazione, già usata, che i punti $x^{30}, x^{2 \mathrm{I}}, x^{\mathrm{r} 2}$ stanno nello $S_{4}$ 2 -osculatore in $x$.

Osserviamo che se la superficie non sta tutta in uno $S_{4}$, caso senza interesse, non possono valere altre equazioni a deriv. parz. terze indipendenti da quelle scritte; in particolare non vi puó essere un'equazione che leghi $x^{0 ;}$ ai punti dello $S_{4}$, e le conseguenze differenziali dell'equazione $(\alpha)$ del $2^{\circ}$ ordine devono essere conseguenze algebriche delle tre ultime.

Derivando $(\alpha)$ rispetto a $\tau_{2}$ e sostituendovi le espressioni delle 3 derivate terze date dalle ( $\beta$ ) si avrebbe un'equazione in $\alpha^{03}$ se $(\alpha)$ contenesse $x^{02}$; poichè ciò non può essere manca $x^{02}$ in $(\alpha)$; c1oè

$$
\left[x^{20}, x^{11}, x^{10}, x^{01}, x\right]=0 .
$$


Analogamente derivando le $(\xi)$ e scrivendo le condizioni d'integrabilità si scorge subito che $x^{02}$ manca anche nelle due prime $(\beta)$, cioè

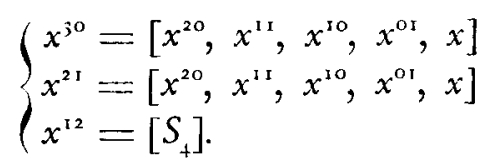

Se $(x)$ contiene $x^{11}$, le $(\beta)$ si scrivono anche

$$
\left\{\begin{array}{l}
x^{30}=\left[x^{20}, x^{10}, x^{01}, x\right] \\
x^{21}=\left[x^{20}, x^{10}, x^{01}, x\right] \\
x^{12}=\left[S_{4}\right]
\end{array}\right.
$$

e confrontando ancora le due espressioni di $x^{31}$ si ottiene una equazione $\mathrm{dcl} 2^{\circ}$ ordine che contiene $x^{02}$ se $x^{30}$ contiene $x^{01}$. Ora se questa non coincide con $(\alpha)$ la superficie sta in $S_{3}$ o è sviluppabile; se invece coincide con $(x) x^{30}$ non puó contenere $x^{0 x}$ e si ha

$$
x^{30}=\left[x^{20}, x^{\mathrm{io}}, x\right]
$$

cioè le curve date $K$ sono piane, e per la seconda delle $(\beta)$, due piani successivi stanno in $S_{3}$ (e per la $3^{\text {a, }}$, tre stanno in $S_{4}$ ).

Se al contrario $(x)$ non contiene $x^{15}$, si riconosce che non puó contenere $x^{\circ}$, cioè $x^{20}=\left[x^{10}, \lambda\right]$ cioè le $K$ sono rette e tre successive stanno in un $S_{4}$, cioè la superficie è rigata d'indice di sviluppabilità $=2$ : ed è noto ${ }^{7}$ ) che le generatrici di una tale rigata si possono considerare tracciate sopra un sistema $\infty^{1}$ di piani, di cui due successivi si tagliano in una retta. Enunciamo la conclusione per $S_{n}$.

Se una superficie di $S_{n}$ possiede un sistema di curve $K$ di cui sia indeterminato il sistema coniugato di $2^{a}$ specie $C$, o la superficie sta in $S_{4}$ oppure le curve $K$ sono piane (in particolare rette) e due piani infinitamente vicini si tagliano in una relta (cioe o sono osculatori ad una curva, o sono tangenti ad un cono di $I^{a}$ specie, o infine passano per una retta fissa).

Ometto di enunciare i risultati, del tutto simili, per il coniugio di specie v.

$$
\$ 8 .
$$

\section{Sistemi coniugati di specie $\vee$ sopra una superficie di $S_{2 \nu+2}$.}

Data una superficie di $S_{2 v+2}$ e su di essa un sistema $\infty^{1}$ di curve $C$ è chiaro che non si puó in generale associarvi un sistema di curve $K$ in modo da ottenere un doppio sistema coniugato di specie $\%$.

7) Cfr. la mia Nota: Alcilne proprietá, etc,; già citata in 3). 
Infatti, riferendosi per cs. al caso $\nu=2$, invece dell'equazione-determinante (I), si avià da annullare una matrice, cioè si hanno due equazioni (in $S_{6}$ ) alle quali non si puó più soddisfare assegnando ad arbitrio uno dei due sistemi di curve (cioè i differenziali $d \circ \delta$ ). Considerate le due equazioni lineari in $\delta \tau_{1} / \delta \tau_{2}$ per la loro compatibilità deve essere-(fatto $d^{2} \tau_{1}=0$ ):

$$
\left|\begin{array}{c}
x \\
x^{10} \\
x^{01} \\
x^{20} d \tau_{1}+x^{11} d \tau_{2} \\
x^{11} d \tau_{1}+x^{02} d \tau_{2} \\
x^{30} d \tau_{1}^{2}+2 x^{21} d \tau_{1} d \tau_{2}+x^{12} d \tau_{2}^{2}+x^{11} d^{2} \tau_{2} \\
x^{21} d \tau_{1}^{2}+2 x^{12} d \tau_{1} d \tau_{2}+x^{03} d \tau_{2}^{2}+x^{02} d^{2} \tau_{2}
\end{array}\right|=0 ;
$$

cioè le $\infty^{5}$ linee $C$ vanno scelte fra le $\infty^{2}$ definite da questa equazione. Poichè il coefficiente di $\left(d^{2} \tau_{2}\right)^{2}$ è $\equiv 0$, si ha:

Sopra una superficie di $S_{6}$ esistono $\infty^{2}$ curve $C$ tali che ad $\infty^{1}$ di esse scelte come curve $C^{(2)}$ ci puo associare un sistema $\infty^{\mathrm{I}}$ di curve $K$ formanti con le prime un doppio sistema coniugato di $2^{a}$ specie $\left(C^{(2)}, K^{(1)}\right)$ : una curva $C$ e univocamente determinata $d a$ un suo punto e dalla tangente ivi.

Cosi si prova che non esistono sistemi coniugati di $2^{2}$ specie sopra una generica superficie di $S_{7}$. Risultato analogo per $\vee>2$.

\section{$\oint 9$.}

\section{Caratterizzazione di superficie in base al coniugio.}

Invertiamo le proprietd trovate per le superficie di $S_{\text {s }}$ e di $S_{6}$ proponendoci i due problemi seguenti ${ }^{\mathbf{8}}$ ):

I) Determinare le $V_{2}$ (di $S_{n}$ ) tali che ad ogni sistema $\infty^{\mathrm{I}}$ di curve $C$ si possa associare un sistema $\infty^{\mathrm{t}}$ di curve $K$ formanti con le prime un doppio sistema $\left(K^{(1)}, C^{(2)}\right)$.

2) Determinare le $V_{2}$ (di $S_{n}$ ) che posseggono $\infty^{2}$ curve tali che assunte $\infty^{\mathrm{t}}$ come

8) Il problema analogo per il coniugio di $\mathrm{I}^{\mathrm{a}}$ specie porta al teorema di SEGRE: la superficie sta in $S_{3}$ o è sviluppabile (in $S_{n},{ }^{\prime} n \gtrsim 3$ ). Alla caratterizzazione dello spazio ambiente o della natura della superficie partendo da proprietà proiettivo differenziali ho già dedicato altri lavori: Sullo spazio d'ımmersione di superficie possedenti dati sistemi di curve [Rendiconti del R. Istituto Lombardo; vol. XLVII ( ${ }^{\circ}$ semestre I9I4, pp. 177-192]; Determinazione delle superficie integrali d'un sistema di equazioni a derivate parziali lineari ed omogenee [ibidem; vol. LII (2o semestre 1919), pp. 6ro-636]; Proprietd diffe. reniall caratteristiche di enth algebrici [Memoric della $R$. Accademia dei Lincei ( $2^{\circ}$ semestre I92 I), in corso di stampal. 
$C$ esista sempre un sistema $\infty^{x}$ di curve $K$ formanti con le prime un doppio sistema $\left(C^{(2)}, K^{(3)}\right)$.

Troveremo oltre alle superficie di $S_{5}$ per I) e di $S_{6}$ per 2) altre classi di superficie che costituiscono una generalizzazione in senso proiettivo ${ }^{9}$ ) delle ordinarie sviluppabili (cfr. $\ 7$ ).

Per il problema I) dobbiamo esprimere che è possibile soddisfare alle equazioni compendiate in

$$
\left|\begin{array}{c}
x \\
x^{\mathrm{oO}} \\
x^{\mathrm{or}} \\
x^{20} d \tau_{1}+x^{1 \mathrm{1}} d \tau_{2} \\
x^{11} d \tau_{1}+x^{02} d \tau_{2} \\
\left(M+x^{11} d^{2} \tau_{2}\right) \delta \tau_{1}+\left(N+x^{02} d^{2} \tau_{2}\right) \delta \tau_{1}
\end{array}\right|=0
$$

(il cui numero dipende dalla dimensione $n$ dell'ambiente $S_{n}$, per ora incognita) qualúnque siano le $C$; e quindi anche, qualunque siano $d \tau_{2} / d \tau_{1}, d^{2} \tau_{2} / d \tau_{1}^{2}$, deve essere identicamente :

$$
\mid \begin{gathered}
x \\
x^{10} \\
x^{01} \\
x^{20} d \tau_{1}+x^{11} d \tau_{2} \\
x^{11} d \tau_{1}+x^{02} d \tau_{2} \\
M+x^{11} d^{2} \tau_{2} \\
N+x^{02} d^{2} \tau_{2}
\end{gathered} \|=0
$$

In particolare, osservando il coefficiente $\mathrm{di} d^{2} \tau_{2}$, deve risultare

$$
\mid \begin{gathered}
x \\
x^{10} \\
x^{01} \\
x^{20} \\
x^{11} \\
x^{02} \\
M d \tau_{1}+N d \tau_{2}
\end{gathered} \|=0
$$

per qualsiasi valore di $d \tau_{2} / d \tau_{1}$.

Se lo spazio 2-osculatore $S(2)$ in un punto generico $x$ (individuato dai punti scritti nelle prime 6 righe della matrice) è uno $S_{S}$ (cioè ha dimensione regolare) tenendo presenti le espressioni di $M$ ed $N$ e l'arbitrarieta di $d \tau_{2} / d \tau_{1}$ si ricava che tutti i punti derivati terzi $\left(x^{30}, x^{21}, \ldots\right)$ stanno in $S_{s}$ : quindi tutta la superficie sta in $S_{s}$.

9) E anche nel senso metrico, per.le applicabilità di specie superiore, specificato nella mia Nota: Problemi movit, etc. [ cià citato in. ${ }^{2}$ ); vad. $\mathrm{n}^{\circ} 5$ ] da cui ha avuto origine questa ricerca. 
Rimane a discutere l'ipotesi opposta: lo $S(2)$ osculatore è un $S_{4}$, o un $S_{3}$ [scartando peró le superficie di $S_{+}$e di $S_{;}$per le quali si perde la nozione di coniugio di $2^{\text {a }}$ specie; si puó scartare anche il casó $S(2) \equiv S_{\text {; }}$ che porta alle ordinarie sviluppabili]. Quando $S(2) \equiv S_{4}$ esiste (sulla superficie un doppio sistema coniugato ordinario o un sistema semplice di asintotiche, quindt) una equazione a derivate parziale del $2^{\circ}$ ordine (soddisfatta dalle $x_{\imath}$ ) in forza della quale la penultima condizione (non l'ultima, che è identicamente soddisfatta) dà luogo ad una equazione a derivate parziali lineare ed omogenea del $3^{\circ}$ ordine: a questa vanno associate le due cquazioni pure del $3^{\circ}$ ordine ottenute derivando quella del $2^{\circ}$; le tre equazioni così ottenute sono linearmente indipendenti, come si verifica subito (perchè contengono derivate terze differenti).

Altre equazioni del $3^{\circ}$ ordine non possono esistere altrimenti la superficie starebbe in $S_{4}$. Sicchè per le superficie in esame in un punto $x$ generico $S(2) \equiv S_{4}$ e $S(3) \equiv S_{5}$. Le uniche superficie di questo tipo sono ${ }^{\text {ro }}$ ): le superficie di $S_{\text {, (con'doppio sistema }}$ coniugato ordinario, o con un sistema di asintotiche); le superficie di $S_{n}(n \geqslant 5)$ con $\infty$ curve piane (eventualmente rette) in $\infty^{\prime}$ piani tali che due infinitamente vicini stanno in uno $S_{3}$. Quindi:

Le superficie tali che ad ogni loro sistema $\infty^{i}$ di curve (C) si puo associare un sistema $\infty^{\mathrm{r}}$ di curve $(K)$ formanti con quelle un doppio sistema coningato $d \imath 2^{a}$ specie sono:

I) le superficie di $S_{s}$;

2) le superficie di $S_{n}(n \doteq 5)$ con $\infty^{\prime}$ curve piane (eventualmente rette) in modo che due piani successivi si taglino lungo una retta, cioe situate:

a) nei piani osculatori ad una curva;

ß) nei piani tangenti ad un cono di $\mathrm{I}^{\mathrm{a}}$ specie;

$\gamma)$ in $\infty^{\mathrm{x}}$ piani per una retta fissa $\left(S_{\mathrm{r}}\right.$-cono).

Passiamo ora a determinare le supetficie di $S_{n}$ ( $n$ per ora incognito) contenenti $\infty^{2}$ curve $C$ tali che ad $\infty^{1}$ di esse si possa sempre associare un sistema $\infty^{\prime}$ di curve $K$ formanti con le prime un doppio sistema $\left(C^{(2)}, K^{(1)}\right)$.

Seguendo il ragionamento di prima si è ancora condotti a scrivere la (2), ma non si csige che sia identicamente soddisfatta: s'impone soltanto che, qualunque sia $d \tau_{2} / d \tau_{1}$, sia possibile ricavare $d^{2} \tau_{2} / d \tau_{1}^{2}$ (il che è evidentemente possibile in $S_{6}$ perchè si ha una sola equazione).

$\left.{ }^{\circ}\right)^{-}$Cfr. Determindzione delle superficie integrali, etc. citata in ${ }^{8}$ ); $\$ 3$, Lemma. Il teorema generale é questo: Se per un punto generico di una $V_{2}$ si ha $S(v) \equiv S_{\rho}$ e $S(v+1) \equiv S_{p+1}$ o la $V_{2}$ sta in $S_{\beta+1} \circ$ contiene $\infty^{\mathrm{I}}$ curve in $S_{\rho-\nu}$ e due $S_{\rho-\nu}$ successivi si tagliano in uno $S_{p-\nu-1}$.

Del resto nel caso attuale si ritrova facilmente il risultato scrivendo l'equazione del $2^{\circ}$ ordine che rappresenta il doppio sistema coniugato o il sistema semplice di asintotiche, ed esamínando (alcune del-) le condizioni d'integrabilità fra questa e la nuova equazione del $3^{\circ}$ ordine. 
Delle due ipotesi $S(2) \equiv S_{5}$ e $S(2) \equiv S_{+}$scurtimo subuto la seconda perchè, come facilmente si verifica, riconduce sui casi precedenti (i quali naturalmente, imponendo più condizioni di quelle in csame ora, si ripresentano come soluzioni particolari; e vanno scartate).

$\dot{\mathrm{E}}$ dunque $\neq 0$ la matrice formata con le coordinate del punto $x$ e dei suoi punti derivati primi e secondi. La condizione di compatibilità delle equazioni precedenti in $d^{2} \tau_{2}$ si scrive

$$
\left|\begin{array}{c}
S(2) \\
x^{30} d \tau_{1}^{2}+2 x^{21} d \tau_{1} d \tau_{2}+x^{12} d \tau_{2}^{2} \\
x^{21} d \tau_{1}^{2}+2 x^{12} d \tau_{1} d \tau_{2}+x^{03} d \tau_{2}^{2}
\end{array}\right|=0
$$

[ove $S(2)$ sta in luogo della matrice ora accennata]. Siegliamo le linee coordinate (fino ad ora arbitrarie) come quelle di un doppio sistema coniugato di $2^{a}$ specie (per ipotesi esistente sulla superficie); varrd un'equazione del tipo

$$
x^{21}=\left[x^{20}, x^{11}, x^{10}, x^{\circ 1}, x\right]
$$

in forza della quale la precedente condizione si scrive

$$
\left|\begin{array}{c}
S(2) \\
x^{30} d \tau_{1}^{2}+x^{12} d \tau_{2}^{2} \\
2 x^{12} d \tau_{1} d \tau_{2}+x^{03} d \tau_{2}^{2}
\end{array}\right|=0 .
$$

Per l'arbitrarietà di $d \tau_{2} / d \tau_{1}$ se ne traggono tre equazioni del tipo

$$
\begin{aligned}
& {\left[x^{30}, x^{12}, S(2)\right]=0} \\
& {\left[x^{30}, x^{03}, S(2)\right]=0} \\
& {\left[x^{12}, x^{03}, S(2)\right]=0 .}
\end{aligned}
$$

Queste non sono certo linearmente indipendenti; se lo fossero la superficie starebbe in $S_{5}$, caso già escluso.

Due di esse peró devono necessariamente essere indipendenti altrimenti la matrice precedente non riuscirebbe $\equiv 0$.

Sicchè per le nostre superficie $S(2) \equiv S_{5}$ e $S(3) \equiv S_{6}\left[\right.$ mentre $S(4) \equiv S_{6}$ o $S_{7}$; per il teorema già ricordato in ${ }^{\text {Io }}$ ) concludiamo:

Le superficie con $\infty^{2}$ curve $C$ tali che ad $\infty^{\prime}$ di esse si possono associare $\infty^{1}$ curve $K$ formanti con le prime un doppio sistema coniugato $\left(C^{(2)}, K^{(1)}\right)$ sono:

I) le superficie di $S_{6}$;

2) le superficie di $S_{n}(n \supseteq 6)$ con $\infty^{\mathrm{x}}$ curve in $\infty^{\mathrm{x}} S_{;}$di cui due successivi si tagliano in un piano, cioè situate; 
*) negli $\infty^{\prime} S_{3}$ osculatori ad una curva;

अ) negli $\infty^{\mathrm{l}} S_{3}$ osculatori ad un cono di $\mathrm{I}^{\mathrm{a}}$ specie;

ү) negli $\infty^{\mathrm{x}} S_{3}$ tangenti ad un cono di $2^{\mathrm{a}}$ specie;

d) in $\infty^{\mathrm{I}} S_{3}$ per un piano $\left(S_{2}\right.$-cono).

Roma, novembre 1921.

EnRICO Bompiani. 